\title{
Wolfgang Plischke
}

Wolfgang Plischke, Bayer's chief of innovation, will be retiring at the end of this month after over 30 years at Bayer. During his time at the German pharmaceutical powerhouse, he has overseen its interests in pharmaceuticals, diagnostics and consumer health, both globally and regionally in North America and Japan. He discusses research and development (RED) funding and the intersection of human, animal and plant research with Asher Mullard.

\section{Q What is your proudest achievement at Bayer?}

I am most proud of what we did from 2002 to 2006 , refocusing our pharma business. We had some issues with our pharmaceutical business after our voluntary withdrawal of Baycol [cerivastatin] in 2001, and had to make significant changes as a result. We created a much more focused specialty pharma organization during these days, focusing on a few indications. Until then we had been active, like most of the bigger pharmaceutical players, in almost any indication. But in 2002 we started focusing on oncology and cardiovascular R\&D.

We also created a new proof-of-concept organization. Like many other research organizations, our development people claimed the research people were just throwing their preclinical development candidates across the fence and saying "I've fulfilled my task; it is now up to you to make a great product out of this". We changed our organization so that the research people had responsibility up to proof-of-concept stages to assure that they had a stake in developing drugs that could be really successful.

On the mechanism side, although we decided to focus on oncology and cardiovascular disease, we also wanted to remain somewhat opportunistic by exploring whether the targets we were working on could also be relevant in other diseases. If we look now at our late-stage pipeline, we have some examples that came from this approach, for example, for macular degeneration.

Looking back, it feels good to see that we made the right decisions.

\section{Q What was your biggest mistake?}

My biggest mistake was not opening up to the external world early enough. When I took over the pharma business in 2001, we were a very internalized organization and still thought that we could do everything ourselves. Now we have changed our approach dramatically, especially in the past 5 years or so. If you look at oncology, we have established a substantial network of collaborators through the whole value chain, from target discovery to clinical trials. Nowadays, collaboration is really a part of our business.

\section{Q Bayer has one of the lowest R\&D budgets} of the top pharma companies ( $€ 3.2$ billion in 2013). Does this put you at a competitive disadvantage?

I am of the belief that you have to limit resources. Yes, you have to have a reasonable spend and you should spend continuously, in bad and good times. But also be radical in your prioritization and don't overspend. If your people don't complain that they don't have enough money, then something is wrong in the organization. We should always have more ideas, and more great ideas, than we have resources. Then the scientists themselves will jump on the most promising projects, which guarantees the right focus. If you have money in excess, everything will be done but it will dilute your efforts. Focus is the key.

\section{Q Does your relatively small R\&D budget create a problem when you think about} collaborating with others?

For sure, it can be easily seen as a threat for our internal scientists who start to worry about which projects will be continued and which will not. But we have put an emphasis on pushing the need for these collaborations because they give us more options.

Q A few large companies have divested or are talking about divesting their animal health units. But in 2011 you took the opposite approach, launching Nimbus to bring your human, animal and plant businesses closer together. What do you hope to achieve with Nimbus? We are the only company in the world that still has health businesses for humans, animals and plants under one roof. And we have quite a history of using the

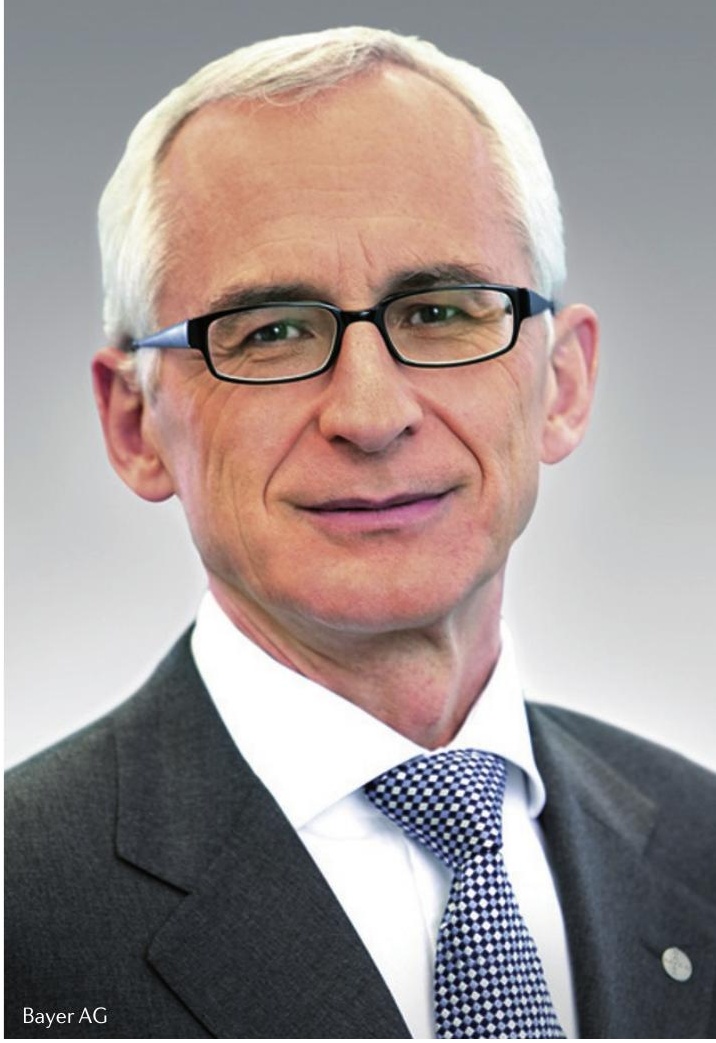

same approaches and similar chemicals in the various businesses. If you look at azoles - fungicides - we have used these in both plant health and pharmaceuticals.

The technologies that our researchers use - high-throughput screening, chemical libraries, genomics databases - are comparable. And the fundamental mechanisms of the molecular and cellular biology are similar in plants and animals and humans. So we set up Nimbus, with a double-digit million-dollar funding, so that more than 100 researchers from across these groups can work together. They are looking into new technology platforms, like protein engineering, systems biology and epigenetics, that we might be able to use more intensively across the business. They are also looking into common molecular mechanisms and targets, like energy metabolism and signalling pathways, that are similar in plants and animals.

This is totally exciting for the organization. It is a motivational culture driver for us, and there is tremendous engagement. We can really move something here. No, we won't have any Phase III drug candidates from this in the next few years. But I believe it will relatively quickly notch up our technology platforms across our businesses. The progress is faster than I expected.

Q What's next for you after retirement? I will go home and have a nice tea with my wife and decide what to do next. But I will still be around in the biochemical industry. 